\title{
Relationship between serum undercarboxylated osteocalcin and hyaluronan levels in patients with bilateral knee osteoarthritis
}

\author{
KIYOHITO NAITO $^{1,2}$, TAIJI WATARI ${ }^{1}$, OSAMU OBAYASHI ${ }^{1}$, \\ SADANOBU KATSUBE $^{3}$, ISAO NAGAOKA ${ }^{4}$ and KAZUO KANEKO ${ }^{2}$
}

\author{
${ }^{1}$ Department of Orthopaedic Surgery, Juntendo University Shizuoka Hospital, Izunokuni, Shizuoka 410-2295; ${ }^{2}$ Department \\ of Orthopaedics, Juntendo University, Bunkyo-ku, Tokyo 113-8421; ${ }^{3}$ Department of Orthopaedic Surgery, Hot Spring of \\ Rehabilitation Nakaizu Hospital, Izu, Shizuoka 410-2502; ${ }^{4}$ Department of Host Defense and Biochemical \\ Research, Juntendo University Graduate School of Medicine, Bunkyo-ku, Tokyo 113-8421, Japan
}

Received November 12, 2011; Accepted December 22, 2011

DOI: 10.3892/ijmm.2012.897

\begin{abstract}
Serum undercarboxylated osteocalcin (s-ucOC) is a marker for vitamin $\mathrm{K}$ metabolism (deficiency). The aim of this study was to investigate the serum levels of ucOC in patients with bilateral knee osteoarthritis (K-OA), and the correlation between ucOC and other biomarkers for K-OA. A total of 25

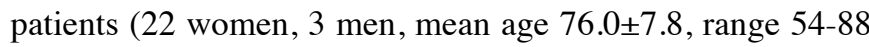
years, mean BMI 24.9 \pm 4.7 ) with a Kellgren-Lawrence grade of 3 or 4 for bilateral knee were enrolled in this study. The levels of s-ucOC and other biomarkers were measured. The levels of s-ucOC $(5.66 \pm 4.70 \mathrm{ng} / \mathrm{ml})$ as well as other cartilage metabolism markers, were elevated in the patients; however, bone metabolism markers were within the normal ranges. Of interest, there was a significant correlation between s-ucOC and serum hyaluronan (a marker for synovitis) $(\mathrm{P}<0.05)$. Our findings suggest that vitamin $\mathrm{K}$ metabolism may be associated with synovitis in patients with $\mathrm{K}-\mathrm{OA}$, and s-ucOC could be a biomarker for K-OA.
\end{abstract}

\section{Introduction}

Osteoarthritis (OA) is a joint disease characterized by pain, cartilage degeneration, and joint stiffness. Although details in the pathogenesis of OA remain elusive, it is clear that this is a complex multifactorial disease process involving cartilage catabolism and anabolism as well as changes in other tissues adjoining joints such as synovium, subchondral bone and tendons (1). Especially, synovitis has more recently gained

Correspondence to: Dr Kiyohito Naito, Department of Orthopaedic Surgery, Juntendo University Shizuoka Hospital, 1129 Nagaoka, Izunokuni, Shizuoka 410-2295, Japan

E-mail: knaito@juntendo.ac.jp

Key words: undercarboxylated osteocalcin, hyarulonan, knee osteoarthritis attention as an important feature in OA (2). As an innervated tissue (3), inflammation of the synovium can be a cause of pain, and synovial effusion has long been known to be a feature of OA.

There are several techniques for detecting the pathophysiological features of OA. Plain radiography provides direct information on bones but not on cartilage, and is characterized by limited sensitivity. Magnetic resonance imaging, which provides direct information on changes in the different joint tissues, is currently being optimized in OA (4). Moreover, many researchers are currently trying to evaluate OA using biomarkers. Biomarkers are molecules derived from the connective tissue matrices, which are released into the systemic circulation and urine during tissue turnover. Several biomarkers have been identified for bone, cartilage, and synovial metabolism in humans, such as serum cross-linked $\mathrm{N}$-terminal telopeptides of type I collagen (s-NTx), serum bone alkaline phosphatase (s-BAP), urinary C-telopeptide degradation products of type II collagen (u-CTX-II), serum C-terminal type II procollagen peptide (s-CPII) and serum hyaluronan (s-HA) (5-7). It has been suggested that biomarkers are useful in identifying those subjects at risk of OA progression and also assessing the therapeutic responses in clinical trials, because the biomarkers are more sensitive than the radiographic change and reliable for measuring the progression (5).

Vitamin $\mathrm{K}$ is an essential cofactor for the post-translational $\gamma$-carboxylation of glutamic acid to form $\gamma$-carboxyglutamic acid (Gla) residues, which confer functionality to the Gla-containing proteins (8). In addition to the coagulation factors, the Gla-containing protein family includes a growth factor Gas-6, a skeletally expressed extracellular matrix protein osteocalcin and a matrix Gla protein (MGP) $(9,10)$. Vitamin K-dependent Gla proteins have high affinity for calcium and phosphate, and thus can bind to the hydroxyapatite crystal surface of mineralized tissues (10). Among Gla-containing proteins, undercarboxylated osteocalcin (ucOC) is regulated by vitamin $\mathrm{K}$ and increases in sera of osteoporosis patients (11). Thus, ucOC is used for a biomarker for vitamin K metabolism (deficiency) (12). Moreover, vitamin K is an important regulator of bone and cartilage mineralization, 
and serum ucOC has been used as an indicator of hip fracture (11). However, ucOC has never been assessed in patients with OA. Thus, in this study, to evaluate ucOC as a marker for $\mathrm{OA}$, we investigated the serum level of ucOC in patients with bilateral knee OA (K-OA), and the correlation of ucOC with other biomarkers for K-OA (s-NTx, s-BAP, u-CTX-II, s-CPII and s-HA).

\section{Materials and methods}

Patients with bilateral knee osteoarthritis. The study was approved by the Ethics Committee for Medical Research of Juntendo University, and informed consent was obtained from all patients.

A total of 25 ( 22 women, 3 men, mean age $76.0 \pm 7.8$, range

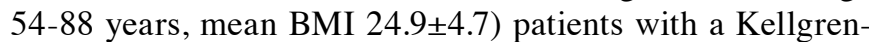
Lawrence $(\mathrm{K} / \mathrm{L})$ grade of 3 or 4 for bilateral knee were enrolled in this study (bilateral grade $3 \mathrm{~K} / \mathrm{L}$ grade total $6, \mathrm{n}=10$; grade 3 and $4 \mathrm{~K} / \mathrm{L}$, grade total $7, \mathrm{n}=7$; and bilateral grade $4 \mathrm{~K} / \mathrm{L}$ grade total $8, \mathrm{n}=8$ ). Patients with knee pain in the medial femorotibial compartment of the studied knee on the day examined, fulfilled the criteria of K-OA of the medial femorotibial joint, as defined by the American College of Rheumatology (ACR) criteria (13). Standing, extended and antero-posterior view and lateral view radiographs were taken at the first visit. An antero-posterior view radiograph was obtained according to the method previously reported (14), and the staging of knee OA based on radiographic examination was assessed using $\mathrm{K} / \mathrm{L}$ grading (15). All radiographs were taken by experienced technicians and scored independently by two readers who were blinded to the clinical information.

Measurement of biomarkers. Both serum and urine samples were obtained from all patients on the day that radiographs were taken. The non-fasted second void urine samples and non-fasted morning blood samples were collected for urine and serum analyses, respectively. The urine and serum samples were stored at $-80^{\circ} \mathrm{C}$ until analysis.

Biomarkers used were s-ucOC, s-NTx, s-BAP, urinary u-CTX-II, s-CPII and s-HA. u-CTX-II values were corrected for urine creatinine concentration $(\mathrm{Cr})$.

All assays including urine creatinine levels were performed with ELISA as follows: s-ucOC (Takara Shuzo Co., Ltd., Kyoto, Japan: intra-assay and inter-assay variation were 4.6 and $5.7 \%$, respectively); u-CTX-II (CartiLaps; Nordic Bioscience, Herlev, Denmark; intra-assay and inter-assay variation were each $<7 \%$ ); s-NTx (Osteomark; OrthoClinical Diagnostics, Rochester, NY; intra-assay and inter-assay variations were $<5$ and $<7 \%$, respectively); s-CP II (IBEX Pharmaceuticals Inc., Montreal, Canada; intra-assay and inter-assay variations were $<10 \%$ ); s-HA and s-BAP (BML Inc., Tokyo, Japan; intra-assay and inter-assay variations were $<5 \%$ and $0.1 \mu \mathrm{g} / 1$, respectively). The measurement of urinary creatinine concentration was performed by a peroxidase-coupled kinetic enzymatic procedure (Kainos, Tokyo, Japan) (16).

Statistical analyses. Data are expressed as mean \pm SD, unless otherwise noted, and analyzed for significant difference by a one-way analysis of variance (ANOVA) with the multiple comparisons test or the Student's t-test (Prism 4, GraphPad
Software, San Diego, CA). Correlation analysis was also performed with Prism 4. Differences were considered statistically significant at $\mathrm{P}<0.05$.

\section{Results}

Measurement of biomarkers. First, we examined the levels of s-ucOC, s-NTx, s-BAP, u-CTX-II, s-CPII and s-HA in bilateral K-OA patients. The levels of $\mathrm{u}-\mathrm{CTX}-\mathrm{II}$, a marker for cartilage type II collagen degradation, $(611.55 \pm 287.24 \mathrm{ng} / \mathrm{mmol}$ Cr), s-CPII, a marker for cartilage type II collagen synthesis, $(922.26 \pm 309.34 \mathrm{ng} / \mathrm{ml})$ and s-HA, a marker for synovitis, $(144.38 \pm 132.35 \mathrm{ng} / \mathrm{ml})$ were elevated in bilateral K-OA patients compared with normal individuals (control range of u-CTXII 340 $\pm 140 \mathrm{ng} / \mathrm{mmol} \mathrm{Cr}$, s-CPII mean $195.99 \mathrm{ng} / \mathrm{ml}$, s-HA $36 \pm 16 \mathrm{ng} / \mathrm{ml})$, as previously reported $(5,7)$. However, the levels of s-NTx (a marker for bone resorption, 15.92 $\pm 5.55 \mathrm{nM} \mathrm{BCE} / \mathrm{l}$ ) and s-BAP (a marker for bone synthesis, 14.85 $\pm 5.45 \mu \mathrm{g} / \mathrm{l}$ ) were within the normal ranges (normal range of s-NTx; male, 9.5-17.7 nM BCE/1, female, 10.7-24.0 nM BCE/1, s-BAP; male, 3.7-20.9 $\mu \mathrm{g} / 1$, female, 3.8-22.6 $\mu \mathrm{g} / \mathrm{l})$. Of interest, s-ucOC (a marker for vitamin $\mathrm{K}$ metabolism, 5.66 $\pm 4.70 \mathrm{ng} / \mathrm{ml}$ ) was also elevated in the OA patients (normal range of s-ucOC; $<4.5 \mathrm{ng} / \mathrm{ml}$ ).

Next, we compared these biomarkers among three groups: $\mathrm{K} / \mathrm{L}$ grade total $6(\mathrm{n}=10), \mathrm{K} / \mathrm{L}$ grade total $7(\mathrm{n}=7)$ and $\mathrm{K} / \mathrm{L}$ grade total $8(n=8)$. There was no significant difference among the three groups in all bone and cartilage biomarkers (NTx, BAP, CTX-II and CPII) (Fig. 1B-E). Of note, s-ucOC and s-HA were marginally increase in $\mathrm{K} / \mathrm{L}$ grade total 8 (s-ucOC, $\mathrm{P}=0.16$ and $\mathrm{s}-\mathrm{HA}, \mathrm{P}=0.28)$ compared with those in $\mathrm{K} / \mathrm{L}$ grade total 6 and 7 (Fig. 1A and F), suggesting that vitamin $\mathrm{K}$ metabolism and synovitis may be associated with the progression of K-OA.

Correlation analysis of the biomarkers in knee OA. As mentioned above, s-ucOC as well as s-CPII, u-CTX-II and s-HA levels was elevated in bilateral K-OA patients. This observation suggests that the s-ucOC level is likely to reflect the cartilage degeneration in K-OA patients, although s-ucOC is known as a marker for vitamin $\mathrm{K}$ metabolism. To evaluate this hypothesis, we analyzed the correlation between s-ucOC and bone metabolism (s-NTx and s-BAP), cartilage metabolism (u-CTX-II, s-CPII) or synovitis (s-HA). Of note, there was a significant correlation between s-ucOC and bone metabolism (s-NTx, $\mathrm{P}<0.01$; s-BAP, $\mathrm{P}<0.01$ ) (Fig. 2). In contrast, the levels of $\mathrm{u}-\mathrm{CTX}$-II (a marker for cartilage degradation) and s-CPII (a marker for cartilage synthesis) were not significantly correlated with s-ucOC (u-CTX-II, P=0.15; s-CPII, P=0.93) (Fig. 3A and B). Of importance, s-ucOC was significantly correlated with s-HA $(\mathrm{P}<0.05)$ (Fig. 3C), suggesting that vitamin $\mathrm{K}$ metabolism may be associated with synovitis in K-OA.

Furthermore, we analyzed the correlations between s-HA and bone metabolism (s-NTx and s-BAP) or cartilage metabolism (u-CTX-II and s-CPII). There was no significant correlation between s-HA and biomarkers for bone and cartilage metabolism (Fig. 4).

These observations support our hypothesis that an association between vitamin $\mathrm{K}$ metabolism and synovitis could be an important feature for OA. 

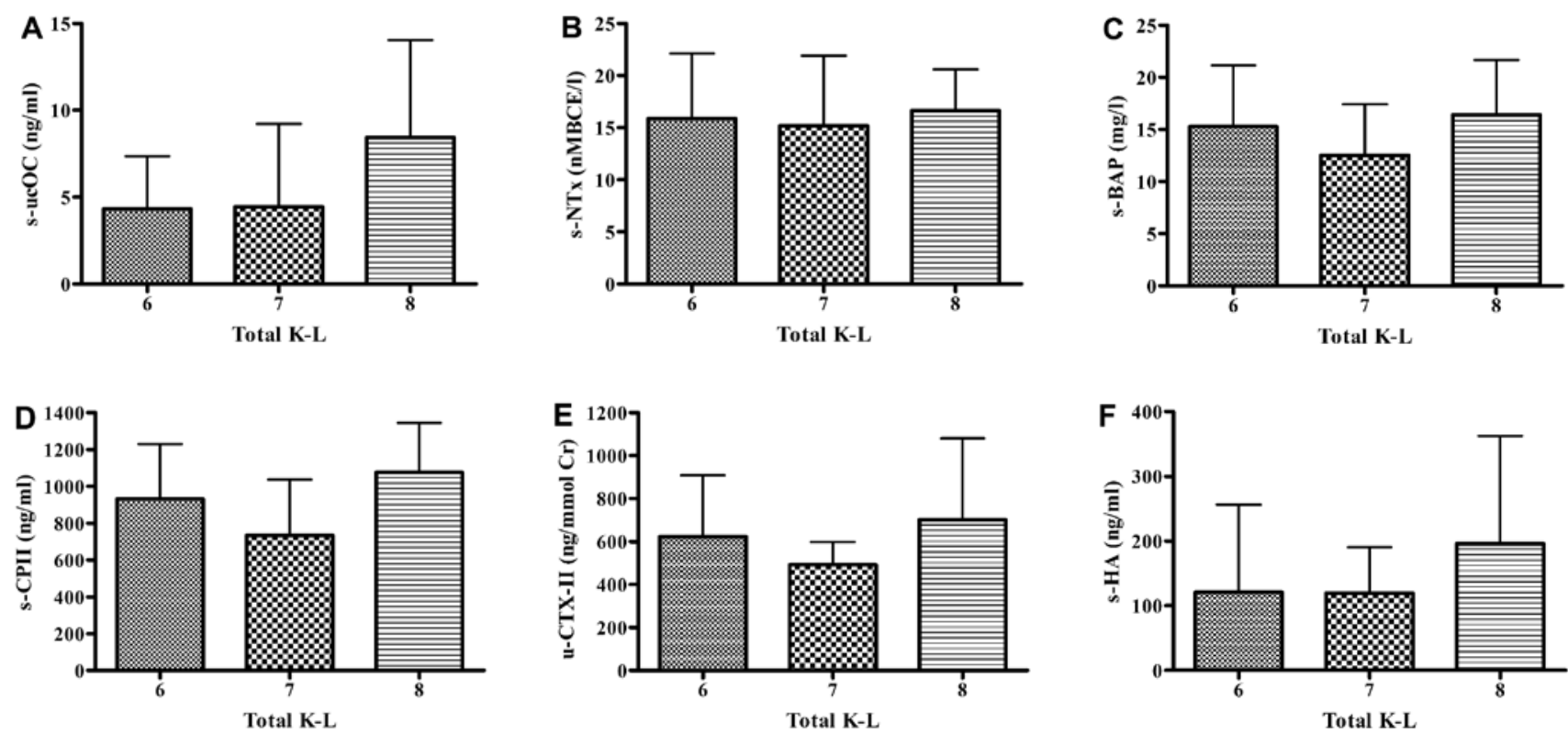

Figure 1. Levels of ucOC, NTx, BAP, CTX-II, CPII and HA in K/L grade total 6, 7 and 8 in patients with bilateral K-OA. Serum levels of (A) ucOC, (B) NTx, (C) BAP, (E) CPII, (F) HA and urinary levels of (D) CTX-II were measured by ELISA. Data represent the mean \pm SD in each group (K/L grade total 6, n=10; grade total 7, $\mathrm{n}=7$; grade total $8, \mathrm{n}=8$ ). Serum (A) ucOC and (F) HA levels were only marginally increase in $\mathrm{K} / \mathrm{L}$ grade total 8 ( $\mathrm{P}=0.16$ and $\mathrm{P}=0.28)$, respectively, compared with that in $\mathrm{K} / \mathrm{L}$ grade total 6 and 7 .
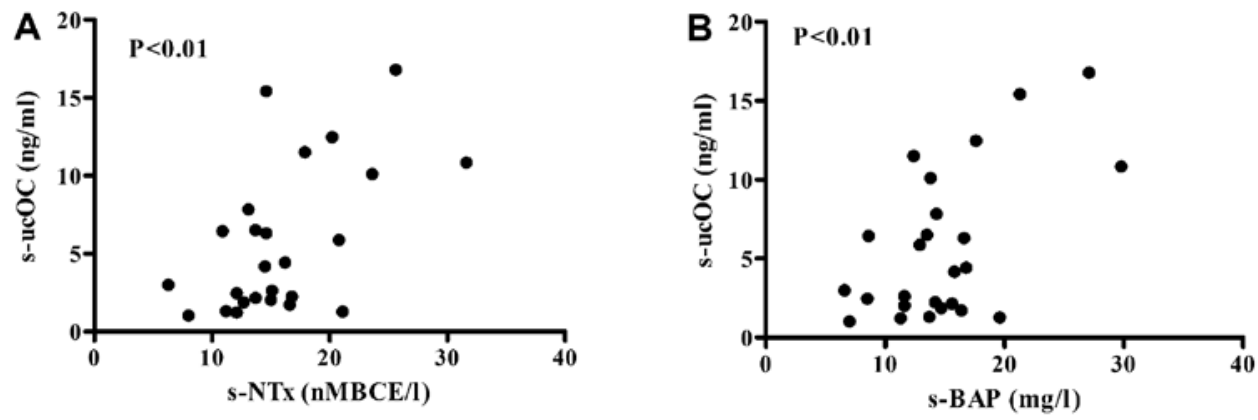

Figure 2. Correlation analysis of ucOC with bone metabolism markers (NTx and BAP) in K-OA. Correlation analysis was performed between (A) s-ucOC and s-NTx, or (B) s-ucOC and s-BAP, using serum samples from patients with bilateral K-OA. P-values are shown in the panels.
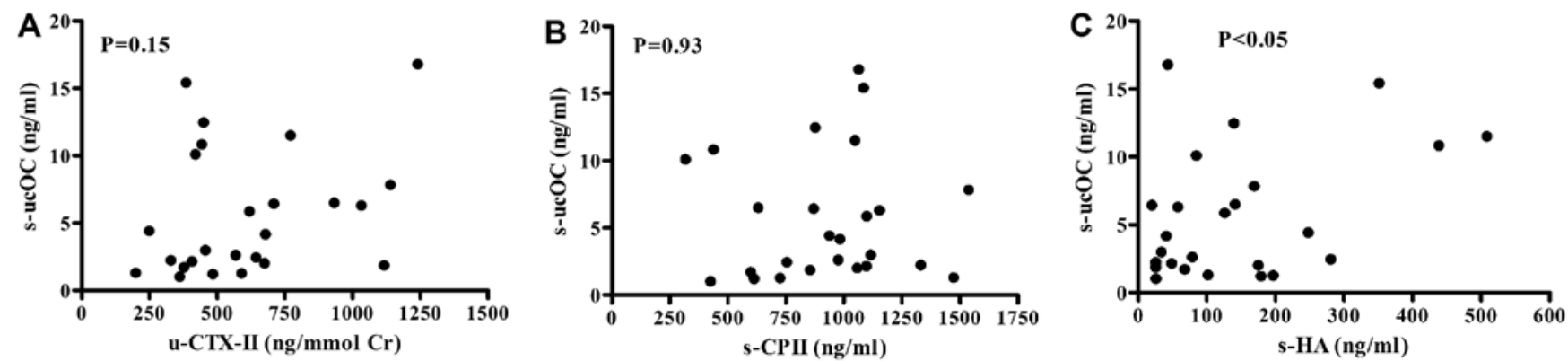

Figure 3. Correlation analysis of ucOC with cartilage metabolism markers (CTX-II and CPII) and synovial metabolism marker (HA) in K-OA. Correlation analysis was performed between (A) s-ucOC and u-CTX-II, (B) s-ucOC and s-CPII, or (C) s-ucOC and s-HA, using urinary and serum samples from patients with bilateral K-OA. P-values are shown in the panels.

\section{Discussion}

Vitamin K has several potential effects on articular cartilage and subchondral bone that may modulate the development of OA (17). Furthermore, inadequate $\gamma$-carboxylation of a growth factor Gas-6, as occurs with insufficient vitamin K, impairs the function of Gas-6 $(9,18)$ and, as such, may impair chondrocyte viability in articular cartilage. In addition, vitamin $\mathrm{K}$ itself suppresses the signaling of certain inflammatory mediators and cytokines, which may be involved in the pathogenesis of OA (19). Taken together, it could be speculated that lack of adequate vitamin $\mathrm{K}$ may be a significant factor in the pathogenesis of 

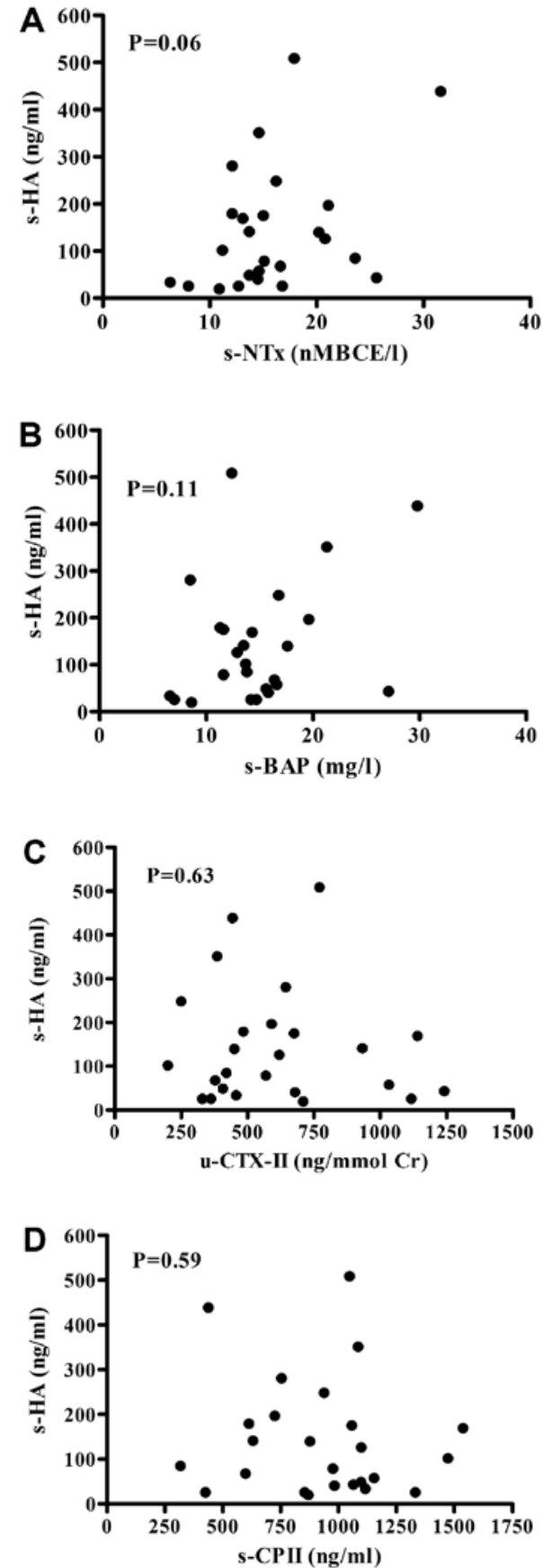

Figure 4. Correlation analysis of HA with NTx, BAP, CTX-II, CPII and HA in K-OA. Correlation analysis was performed between (A) s-HA and s-NTx, (B) s-BAP, (C) u-CTX-II or (D) s-CPII using serum and urinary samples from patients with bilateral K-OA. P-values are shown in the panels.

OA, with particular effects on the osteophyte development and joint space narrowing, which reflect cartilage loss.

As a major product of synovial cells, HA is recognized as a marker for synovitis. HA levels are increased in patients with rheumatoid arthritis (RA), and the highest levels have been found in patients with aggressive RA of both large (hip and knee) and small (hands and feet) joints (20). Although lower than in RA, the increased levels of HA are also found in patients with knee OA, and their levels are higher in the accelerated disease and cartilage destruction (21). Thus, the serum HA level could be a potential prognostic marker for the joint destruction in RA and OA.
In this study, we assessed the serum and urinary biomarkers for bone, cartilage and synovial metabolism in patients with bilateral K-OA, who had a K/L grade of 3 or 4 for bilateral knee. Roberts et al (22) have reported that vitamin K concentration is low in osteoarthritis patients, but they have not mentioned the relationship between vitamin $\mathrm{K}$ concentration and cartilage metabolism. In this regard, this is the first study to consider this relationship in patients with bilateral K-OA. We confirmed that u-CTX-II, s-CPII and s-HA levels were elevated in K-OA compared with normal individuals, indicating the association of increased cartilage turnover and synovitis in K-OA. In contrast, the level of s-NTx was not increased in K-OA in this study, although the s-NTx levels are reportedly increased in patients with OA (6). Thus, the change of bone metabolism, as assessed with s-NTx, in OA remains controversial. Of note, the levels of s-ucOC, a marker for vitamin $\mathrm{K}$ metabolism (deficiency), was increased in K-OA, suggesting that vitamin K may be partly deficient in patients with K-OA. Moreover, s-ucOC and s-HA were marginally increased in $\mathrm{K} / \mathrm{L}$ grade total 8 (s-ucOC, $\mathrm{P}=0.16$ and s-HA, $\mathrm{P}=0.28$ ) compared with that in $\mathrm{K} / \mathrm{L}$ grade total 6 and 7 (Figs. 1A and 2A), although u-CTX-II and s-CPII were not increased in $\mathrm{K} / \mathrm{L}$ grade total 8 compared with those in $\mathrm{K} / \mathrm{L}$ grade total 6 and 7 (Fig. 2B and C). These observations likely suggest that vitamin $\mathrm{K}$ metabolism (as evaluated by s-ucOC) and synovitis (as evaluated by s-HA) may participate in the progression of K-OA. In addition, there was a significant correlation between s-ucOC and s-HA levels (Fig. 4C). Together these observations suggest that the possible association of vitamin $\mathrm{K}$ metabolism (as assessed by s-ucOC) and synovitis (as assessed by s-HA) may provide novel insight into the pathogenesis of secondary OA, which is caused by the multiple factors related with lifestyle (such as malnutrition) and aging diseases (such as osteoporosis).

However, this study has some limitations. First, the investigation is a single-arm study and not a randomized trial. Therefore, the design may have introduced certain bias into the results. Second, the serum and urine sample collections were not timed or fasted, although diurnal variation of some biomarkers has been reported $(23,24)$. Meanwhile, the collection of second void urine samples is recommended (24), as performed in this study. Third, only Japanese patients are analyzed in the present study. Consequently, our findings cannot be generalized to other ethnic groups. Fourth, the contribution of other joints, which are not conducted in this study, to the levels of biomarkers cannot be ruled out. In addition, the correlation between the degree of radiological change and symptoms is weak, and it is common for patients with radiological osteoarthritis to have few or no symptoms, whereas classical symptoms of osteoarthritis may occur in the absence of structural changes on plain radiography (1). Because the diagnostic criteria for OA are not well defined, it is difficult to set up a control group.

The present study suggests a possibility that not only synovitis but also vitamin $\mathrm{K}$ metabolism could be a factor for developing K-OA, and s-ucOC may be a useful marker for evaluating the pathophysiological condition of K-OA.

\section{Acknowledgements}

We thank Mr. Kouhei Yuasa (BML Inc., Tokyo, Japan) for technical expertise. 


\section{References}

1. Lohmander LS: What can we do about osteoarthritis? Arthritis Res 2: 95-100, 2000

2. Ayral X, Pickering EH, Woodworth TG, Mackillop N and Dougados M: Synovitis: a potential predictive factor of structural progression of medial tibiofemoral knee osteoarthritis e results of a 1 year longitudinal arthroscopic study in 422 patients. Osteoarthritis Cartilage 13: 361-367, 2005.

3. Mapp PI: Innervation of the synovium. Ann Rheum Dis 54: 398-403, 1995.

4. Gray ML, Eckstein F, Peterfy C, Kim YJ and Sorensen AG: Toward imaging biomarkers for osteoarthritis. Clin Orthop Relat Res 427 (Suppl): S175-S181, 2004.

5. Garnero P, Rousseau JC and Delmas PD: Molecular basis and clinical use of biochemical markers of bone, cartilage, and synovium in joint diseases. Arthritis Rheum 43: 953-968, 2000.

6. Bettica P, Cline G, Hart DJ, Meyer J and Spector TD: Evidence for increased bone resorption in patients with progressive knee osteoarthritis. Arthritis Rheum 46: 3178-3184, 2002.

7. Kim TH, Stone M, Payne U, Zhang X, Ionescu M, Lobanok T, King L, Poole AR and Inman RD: Cartilage biomarkers in ankylosing spondylitis relationship to clinical variables and treatment response. Arthritis Rheum 52: 885-891, 2005.

8. Furie B, Bouchard BA and Furie BC: Vitamin K-dependent biosynthesis of $\gamma$-carboxyglutamic acid. Blood 93: 1798-1808, 1999.

9. Loeser RF, Varnum BC, Carlson CS, Goldring MB, Liu ET, Sadiev S, Kute TE and Wallin R: Human chondrocyte expression of growtharrest-specific gene 6 and the tyrosine kinase receptor axl: potential role in autocrine signaling in cartilage. Arthritis Rheum 40: 1455-1465, 1997.

10. Price PA: Gla-containing proteins of bone. Connect Tissue Res 21: 51-57, 1989.

11. Miura M: Biochemical markers of bone turnover. New aspect. An automated assay for measuring bone turnover markers. Clin Calcium 19: 1160-1169, 2009.

12. Liu G and Peacock M: Age-related changes in serum undercarboxylated osteocalcin and its relationships with bone density, bone quality, and hip fracture. Calcif Tissue Int 62: 286-289, 1998 .

13. Altman R, Asch E, Bloch D, Bole G, Borenstein D, Brandt K, Christy W, Cooke TD, Greenwald R, Hochberg M, et al: Development of criteria for the classification and reporting of osteoarthritis. Classification of osteoarthritis of the knee. Diagnostic and Therapeutic Criteria Committee of the American Rheumatism Association. Arthritis Rheum 29: 1039-1049, 1986.
14. Ravaud P, Auleley GR, Chastang C, Rousselin B, Paolozzi L, Amor B and Dougados M: Knee joint space width measurement: an experimental study of the influence of radiographic procedure and joint positioning. Br J Rheumatol 35: 761-766, 1996.

15. Kellgren JH and Lawrence JS: Radiological assessment of osteoarthrosis. Ann Rheum Dis 16: 494-502, 1957.

16. Goren MP, Osborne S and Wright RK: A peroxidase-coupled kinetic enzymatic procedure evaluated for measuring serum and urinary creatinine. Clin Chem 32: 548-551, 1986.

17. Neogi T, Booth SL, Zhang YQ, Jacques PF, Terkeltaub R, Aliabadi P and Felson DT: Low vitamin K status is associated with osteoarthritis in the hand and knee. Arthritis Rheum 54: 1255-1261, 2006.

18. Nakano T, Kawamoto K, Kishino J, Nomura K, Higashino K and Arita H: Requirement of $\gamma$-carboxyglutamic acid residues for the biological activity of Gas6: contribution of endogenous Gas6 to the proliferation of vascular smooth muscle cells. Biochem J 323: 387-392, 1997

19. Hara K, Akiyama Y, Tajima T and Shiraki M: Menatetrenone inhibits bone resorption partly through inhibition of PGE2 synthesis in vitro. J Bone Miner Res 8: 535-542, 1993.

20. Mansson B, Carey D, Alini M, Ionescu M, Rosenberg LC, Poole AR, Heinegård D and Saxne T: Cartilage and bone metabolism in rheumatoid arthritis: differences between rapid and slow progression of disease identified by serum markers of cartilage metabolism. J Clin Invest 95: 1071-1077, 1995.

21. Sharif M, George E, Shepstone L, Knudson W, Thonar EJ, Cushnaghan J and Dieppe P: Serum hyaluronic acid level as a predictor of disease progression in osteoarthritis of the knee. Arthritis Rheum 38: 760-767, 1995.

22. Roberts NB, Holding JD, Walsh HP, Klenerman L, Helliwell T, King D and Shearer M: Serial changes in serum vitamin K1, triglyceride, cholesterol, osteocalcin and 25-hydroxyvitamin D3 in patients after hip replacement for fractured neck of femur or osteoarthritis. Eur J Clin Invest 26: 24-29, 1996.

23. Criscione LG, Elliott AL, Stabler T, Jordan JM, Pieper CF and Kraus VB: Variation of serum hyaluronan with activity in individuals with knee osteoarthritis. Osteoarthritis Cartilage 13: 837-840, 2005.

24. Kong SY, Stabler TV, Criscione LG, Elliott AL, Jordan JM and Kraus VB: Diurnal variation of serum and urine biomarkers in patients with radiographic knee osteoarthritis. Arthritis Rheum 54: 2496-2504, 2006. 